\title{
THE IRONIES OF HUMAN MIND: A CASE OF RETT SYNDROME
}

\author{
Sayan Chattopadhyay ${ }^{1}$, Rachita Arora ${ }^{2}$
}

\begin{abstract}
BACKGROUND: Rett Syndrome (RS) is a chromosome X-linked genetic neurological disorder characterized by developmental regression, particularly in relation to expressive language and use of the hands. It is also associated with profound mental retardation and almost exclusively affects females.

CASE DETAILS: A four and a half year old girl reported to our dental OPD for a dental checkup. On complete examination, she was diagnosed to be suffering from Rett Syndrome. Preventive therapies and proper oral hygiene instructions were explained to her mother.

CONCLUSION: Early diagnosis of such disorders is extremely important along with treatment of patients' problems with love and care to prevent them from further pain and stress. KEYWORDS: Rett Syndrome, stereotypic hand movements, behavior therapy.
\end{abstract}

\section{DOI: http://dx.doi.org/10.4314/ejhs.v24i2.10}

\section{INTRODUCTION}

There is no enigma like the mind. The normal mind functions at the speed of light, in a multitude of directions, and yet, there are certain shadows that are never dissolved by light and remain a deep mystery, as when presented by a disordered human mind. This case report is an attempt to present one of such numerous mysteries that the mind holds, a suspected case of Rett Syndrome (RS).

Rett syndrome is a pediatric neurological development disorder characterized by the regression of psychomotor development with autistic manifestations, decelerated growth of head, seizures, and stereotyped repetitive movements of the hands (1). First described in 1966 by Andreas Rett, an Austrian Physician, and thereafter by Bengt Hagberg et al. in 1983, this syndrome was shown in 1999 to be caused primarily by mutations in the $\mathrm{X}$-linked gene MECP2 [encoding MeCP2 (Methyl CPGbinding protein 2)] protein 2 (2).

The estimated prevalence of the disorder ranges from 1 in 10000 to 1 in 22000. Despite reports from all over the world, very few cases have been reported from the Indian subcontinent. This may probably be due to the under diagnosis of this condition or misdiagnosis of affected children as cerebral palsy or autism (2). Originally, the condition was thought to be confined only to girls, but boys with features close to this disorder have also been described (3).

Girls with RS are born apparently healthy with normal prenatal and perinatal periods. Their psychomotor development is normal up to $1^{\text {st }}$ or $2^{\text {nd }}$ year of life when brain functional regression begins. The regression is clinically manifested with a loss of manual motor skills and appearance of stereotyped movements, gait apraxia, loss of verbal and nonverbal communication skills and association of episodes of hyperventilation, aerophagia and epileptic seizures (1).

Not many case reports have been published in this regard. This case report is an effort to present and highlight the basic oral problems of such children, the criteria for diagnosing the disorder and the strategies employed to prevent and manage their oral problems.

\footnotetext{
${ }^{1}$ Department of Oral Medicine \& Radiology, Awadh Dental College, Jamshedpur, Jharkhand

${ }^{2}$ Department of Pedodontics \& Preventive Dentistry, Aw adh Dental College, Jamshedpur, Jharkhand

Corresponding Author: Rachita Arora, Email: rachita.arora1@gmail.com
} 


\section{CASE DESCRIPTION}

A girl aged four and a half years old who visited the OPD with her mother complained of decayed upper front teeth since last 6 months.

Birth and Development History: The child's mother gave history of normal delivery of the child and confirmed that she did not suffer any medical condition or take any sort of medication during her pregnancy. At birth, her child's height and weight were normal. Gross motor, fine motor, social and emotional and language milestones were also normal during the first one and a half years of her life. After that, the child's milestones got delayed and her development slowed down to the extent of regression.

Clinical Features: The girl presented with a unique feature of continuous stereotypic hand movements (Fig 3). She clenched her fingers so tight that wounds and wasting could be observed on her phalangeal joints (Fig 2). She was unable to perform hand functions like grasping, touching and holding objects.

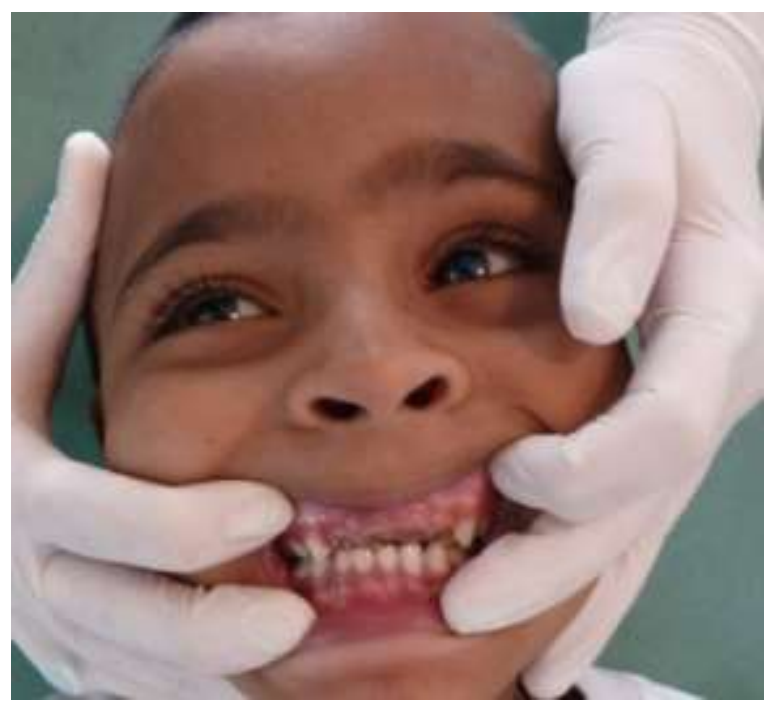

Figure 1: Decayed upper incisors of the girl

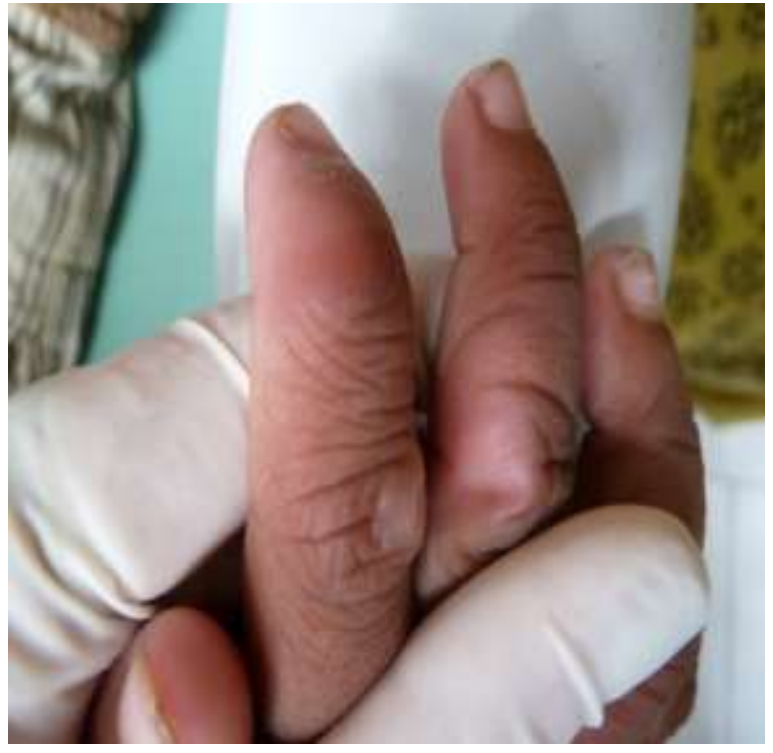

Figure 2: Lesions on fingers caused due to stereotypic hand movements.

The child could not speak any meaningful words and her speech was only limited to a few sounds. Her history revealed that she was able to speak better in the first three years of her life.

The child could not sit still nor could she make an eye contact with the operator. Her gait was abnormal and slow-she needed support to walk. She looked weak, and according to her mother, was unable to eat much due to poor control on her tongue and felt difficulty in swallowing.

The child was unable to understand and comprehend instructions and exhibited autism. Though children with RS may exhibit seizures in late stages (4), the mother of this child gave no history of any such episode.

Intraoral examination of the child revealed four grossly decayed maxillary incisors (Fig 1). Other than that, there were no significant findings. The mother gave recent history of involuntary teeth grinding by the child but no significant attrition was observed.

Treatment: On first visit, mouth prop was used for exploration of the dentition and gross excavation of the carious lesion was done. 


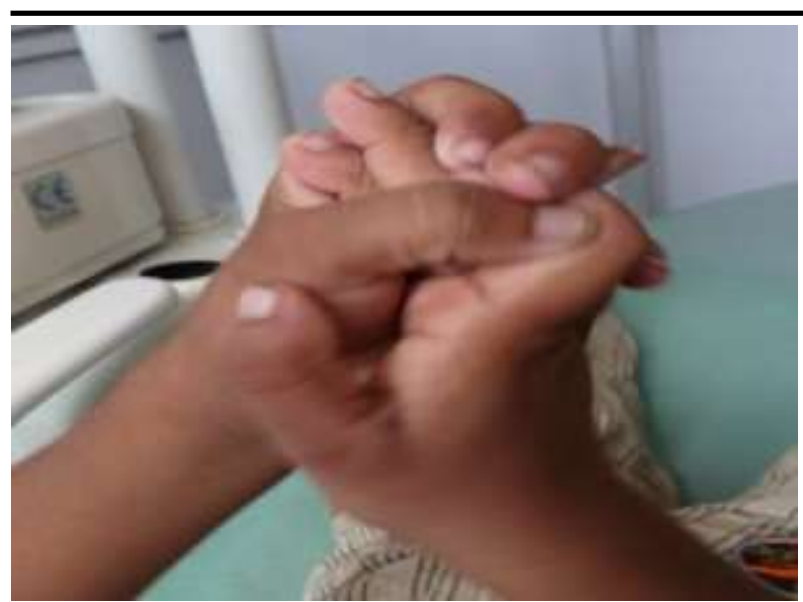

Figure 3: Stereotypic hand movements performed by the child

The mother of the child was told about the possible neurological problem of the child and was advised to get neurological consultation for her daughter. She was counseled to emphasize the importance of primary dentition and prevention of further oral disease. She was given instructions on more use of non-cariogenic diet for the girl, daily application of fluoride gel or mouth rinses. The mother was requested to give frequent follow up visits with her child so that the child could get accustomed to the dental environment and be more cooperative during dental treatment. She was also told about fabrication of a soft splint if the habit of grinding progressed.

Treatment of such children requires the use of sedation or general anesthesia. Unfortunately the anterior teeth could not be treated as the mother of this child was not ready for the advised treatment.

\section{DISCUSSION}

Two groups of oral manifestations of Rett syndrome can be distinguished: those derived from the medication used to treat the disease and those derived from the condition of the syndrome itself.

The drug-related (anxiolytic and anticonvulsant) orofacial manifestations of RS are: xerostomia, stomatitis, glossitis, erythema multiformae, gingivitis, periodontal abscess, sinusitis, dysphagia and tongue paralysis. The oral manifestations of the syndrome itself are not exclusive of the syndrome, since they can also be seen in excessive oral/digital habits, difficulties for correct oral hygiene, etc. However, features like bruxism, anterior open bite, high-arched palate and gingivitis are more prevalent (1).
Other common problems associated with RS are presence of scoliosis (5), constipation (6), gall bladder problems (6), arrhythmias (7) and sleep problems (8). The course of RS is divided into four basic stages $(9,10)$. These are:

Early onset phase (6-18 months of age): unnoticeable slowing of development.

Rapid Destructive phase (1-4 yrs): The child loses skills quickly. Voluntary hand movements and speech are usually first skills lost. Difficult breathing and stereotypic hand movements may start. Social aloofness begins and females demonstrate autistic traits.

Plateau Phase (2-10 yrs): The regression slow s and the condition of child improves. Involuntary movements persist. Seizure activity is prominent and females have improved alertness.

Late Motor Deterioration Phase: This lasts for decades. The individual becomes stiff and may lose muscle tone. Severe scoliosis may occur.

Use of splints is controversial in Rett Syndrome to treat bruxism. Few authors contraindicate the use of splints due to mental retardation and deficient oro motor skills of the child while others like Alpoz et al. have treated bruxism with soft splints, and special designs to avoid anterior mandibular movement accompanying bruxism $(1,11)$.

In the present case, bruxism was not very evident in the anterior teeth as it is in most of the cases of RS. The probable reason for the absence of the feature must be the young age of the girl and her decayed anterior maxillary teeth. Such cases, however, should be encouraged for regular follow up for prophylactic care and checkups of dental and jaw development.

RS is most often mistaken as autism, cerebral palsy, or nonspecific developmental delay. In the past, the diagnosis required numerous diagnostic tests and procedures to rule out other disorders. However, since the discovery of MECP2 gene, a blood test can check whether a child has a gene mutation that causes RS. The sole presence of this mutation is not enough to confirm RS; therefore, clinical features and history of the child cannot be ignored (10).

The self-injurious behavior of the affected children has been intervened using splints for the elbow (elbow orthosis). Katisyannis et al. reported in a study that most successful intervention for selfinjurious behavior was differential reinforcement paired with response interruption.

The behavioral management of the syndrome deals with: 
a) Speech language and communication therapy (13) which includes:

i) Lovaas Method / Applied Behavior Analysis (1987)-The trainer models the child a skill to master and encourages him to replicate. Operant conditioning principles are used to teach language with positive reinforcement.

ii) Treatment and education of autistic and related communication handicapped children (TEACCH) (1970)-This focuses on teaching the child communication skills versus speech skills.

iii) Play Therapy (1997)-This one promotes skills designed to facilitate positive social interactions, either verbal/non verbal using play designs.

iv) Option method / Son-rise program (1970)This is a home based, child -centered approach that encourages the parents to participate and motivate the child. (4)

v) Augmentative and Alternative Communication (1996)-This approach can be very beneficial when dealing with $\mathrm{RS}$ affected children. Such strategy includes eye gaze and voice output devices. Their use can help in effective communic ation.

\section{b) Physical Therapy $(13,14)$}

According to the International Rett Syndrome Association, physical therapy should be one of the priorities in RS patients. Stage 1 should focus on independent sitting, standing and walking; stage 2 on range of motion and ambulation. Stage 3 should emphasize sitting, and stage 4 requires all aspects from previous stages.

\section{c) Occupational the rapy (14)}

It should focus on hand use and eating skills. The child should be assisted with adaptive eating utensils and try to feed independently. Sensory integration therapies, such as tactile activities and joint compression can help the child to interpret and respond better.

The supportive therapies include Music Therapy (2001) and Hydro therapy (2003) (13).

Rett Syndrome is unique in its own way; so are the affected children. These children need early diagnosis and various medical and non-medical therapies to relieve their symptoms. Besides therapy, they need the love and affection of people around them. Therefore, it is very important to help in diagnosing such children, counseling their parents and trying to establish communication with them so that they allow us to treat them and prevent their oral problems as much as possible.

\section{REFERENCES}

1. Gonzalez MCF, Silvestre FJ and Silla JMA (). Oral findings in Rett syndrome: A systematic review of dental literature. Med Oral Patol Oral Cir Bucal, 2011;16 (1): e37-41.

2. Kumar S, Alexander M and Gnanamuthu. Recent experience with Rett syndrome at a tertiary care center. Neurology India, 2004;52 (4): 494-495.

3. Bathla M, Chandna S and Bathla JC. Rett's Syndrome: Diagnostic and therapeutic dilemma. German J Psychiatry, 2010;13(3):157.

4. Glaze D, Schultz R and Frost J (). Rett syndrome: Characterization of seizures and non-seizures. Electroencephalography and Clinical Neurophysiology, 1998;106: 79-83.

5. Kerr AM, Webb P, Prescott RJ, Milne Y. Results of surgery for scoliosis in Rett Syndrome. Journal of Clinical Neurology, 2003; 18: 703-708.

6. Percy AK and Lane JB. Rett syndrome: Clinical and molecular update. Current opinions in Pediatrics, 2004; 16: 660-677.

7. Guideri F, Acampa M, Diperri T, Zapella M, Hayek Y. Progressive cardiac dysautonomia observed in patients affected by classic Rett Syndrome and not in the preserved speech variant. Journal of Child Neurology, 2004;16: 370-373.

8. Nomura Y. Early behavior characteristics and sleep disturbance in Rett syndrome. Brain and Development, 2005; 27 (S1): S35-S42.

9. Hagberg B, Hanefiled F, Percy A, Skjeldal O. An update on clinically applicable diagnostic criteria in Rett syndrome. Comments to Rett syndrome clinical criteria consensus panel satellite to European Pediatric Neurology Society Meeting, Germany. European Journal of Pediatric Neurology, 2001;6: 293-297.

10. Beisang A, Tervo $M$ and Wagner $R$. Rett syndrome: Infancy to Adulthood. A Pediatric Perspective, 2008; 17 (1): 1-3

11. Alpoz AR, Ergul $\mathrm{N}$ and Oncag O. Bruxism in Rett syndrome: a case report. J Clin Ped Dent, 199; 23: 161-163.

12. Katsiyannis A, Ellenburg JS, Acton OM, Torrey G. Addressing the needs of students with Rett syndrome. Teaching Exceptional Children, 2001;33 (5): 74-78.

13. Aithal S, Manjunath YN and Shyamala KC. Rett syndrome: A case study of Malayalam speaking children with an Autism Spectrum Disorder. Language in India, 2011; 4: 304-321.

14. International Rett Syndrome Association (2005). Rett syndrome: Rosetta stone of neurologic diseases. Retrieved April 15, 2005, from http://www.retts yndrome.org/main/update-on-rettsyndrome.htm 